\title{
The dynamical ages of two low-luminosity young radio sources
}

\author{
N. de Vries ${ }^{1}$, I. A. G. Snellen ${ }^{1}$, R. T. Schilizzi ${ }^{1,2}$, and K.-H. Mack ${ }^{3}$ \\ 1 Leiden Observatory, Leiden University, PO Box 9513, 2300 RA Leiden, The Netherlands \\ e-mail: vriesn@strw. leidenuniv.nl \\ 2 Square Kilometre Array Program Development Office, Jodrell Bank Centre for Astrophysics, Manchester M13 9PL, UK \\ 3 Istituto di Radioastronomia - INAF, Via P. Gobetti 101, 40129 Bologna, Italy
}

Received 16 June 2009 / Accepted 1 June 2010

\begin{abstract}
Context. Young radio-loud active galactic nuclei form an important tool for investigating the evolution of extragalactic radio sources. To study the early phases of expanding radio sources, we are using the CORALZ sample, which contains $25 \operatorname{compact}\left(\theta<2^{\prime \prime}\right)$ radio sources associated with nearby galaxies.

Aims. In this paper we aim to determine the lobe-to-lobe expansion speeds for two sources in the sample. Comparing the dynamical ages of these relatively low-luminosity objects with ages of powerful sources found in the literature, will serve to test radio source evolution models.

Methods. We present our third epoch of VLBI observations, using the EVN+VLBA array at $5.0 \mathrm{GHz}$, at four and eight years after our previous measurements.

Results. We report the detection of a lobe-to-lobe expansion speed of $0.095 \pm 0.020 \mathrm{~h}^{-1} \mathrm{c}$ for CORALZ J083139+460800, and a $1-\sigma$ upper limit to the expansion speed of CORALZ J131739+411545 of $0.074 \mathrm{~h}^{-1} \mathrm{c}$. The detected expansion speed of CORALZ J083139+460800 corresponds to a dynamical age of $245 \pm 55$ years.

Conclusions. The detected expansion speed and upper limit are lower than any presented in the literature for powerful young AGN. This confirms that the expansion speeds of young radio sources are correlated with their radio luminosities.
\end{abstract}

Key words. galaxies: active - radio continuum: galaxies

\section{Introduction}

Young radio-loud active galactic nuclei (AGN) are key objects for constraining evolution models of classical double radio sources. Young radio sources can be recognised by their small sizes, by their inverted spectra at low frequencies, and by their often compact symmetric morphologies, with lobe-like structures on both sides of a central core. Depending on the observed characteristics, they are called compact steep spectrum (CSS) sources (Peacock \& Wall 1982; Fanti et al. 1990; O'Dea 1998), gigahertz peaked spectrum (GPS) sources (O’Dea 1998), and/or compact symmetric objects (CSO, Wilkinson et al. 1994; Conway 2002).

Samples of young radio-loud AGN have been used in a statistical way to constrain the early evolution of radio sources. Relative number statistics indicate that at a young age, radio sources must have significantly higher radio luminosities than when they are older (O'Dea \& Baum 1996; Readhead et al. 1996; Fanti \& Fanti 2003). Some analytic models predict that this decrease in radio luminosity is preceded by a period of increasing luminosity at the earliest evolutionary stages (Snellen et al. 2000, 2003).

Very important results come from multi-epoch Very Long Baseline Interferometry (VLBI) observations of individual CSOs. For a handful of powerful objects, the proper motions of the lobes have been detected, from which their dynamical ages can be estimated (Owsianik \& Conway 1998; Polatidis \& Conway 2003, and references therein). Separation velocities between the opposite extremities of the sources of up to $0.5 \mathrm{~h}^{-1} \mathrm{c}$ have been measured, corresponding to dynamical ages in the range of a few hundred to a few thousand years.

Snellen et al. (2004) selected a sample of 25 young radio sources, CORALZ (COmpact RAdio sources at Low Redshift), with the aim of obtaining an unbiased view of young radio-loud AGN in the nearby universe. The sources in the CORALZ sample are significantly closer than the archetypal CSO and GPS sources studied in the literature. Proper motions should be easier to detect in these nearby sources, since similar intrinsic expansion velocities would result in significantly higher angular motions than in previous studies. Moreover, in general these radio sources are less powerful than the archetypal CSOs and GPS sources, which will allow luminosity-dependent comparison studies.

The CORALZ sample was selected on flux density $\left(S_{1.4 \mathrm{GHz}}>100 \mathrm{mJy}\right)$ and angular size $\left(\theta<2^{\prime \prime}\right)$. Using the Very Large Array (VLA) Faint Images of the Radio Sky at Twenty-centimeters (FIRST) survey (White et al. 1997), the optical Automated Plate Measuring machine (APM) catalogue of the first Palomar Observatory Sky Survey (POSS-I) (McMahon \& Irwin 1991), and follow-up observations, all radio sources identified with bright galaxies were selected (red magnitude of $e<16.5$ mag or blue magnitude of $o<19.5 \mathrm{mag}$ ). A detailed description of the selection process and additional radio observations can be found in Snellen et al. (2004). Nearly all of the sources in the CORALZ sample are spectrally classified as GPS and CSS sources, and morphologically classified as CSOs, confirming that these are likely to be young radio sources. 
In our previous paper (de Vries et al. 2009) we presented radio maps, morphological classifications, and linear sizes of the CORALZ sample. Furthermore we derived a remarkably low upper limit to the expansion speed of one of the sources (CORALZ J083139+460800, which is re-examined in this paper). Since the CORALZ sample is of relatively low luminosity, we interpreted this as a hint that the expansion speeds of young radio sources are correlated with their radio luminosities. We now wish to investigate this further.

In this paper we present a new epoch of global VLBI observations for two sources from the CORALZ sample. For these two radio sources, for which in total three epochs of VLBI observations have been obtained with the same telescope array at the same frequency, we will estimate expansion velocities. The new observations of the other sources from the CORALZ sample will be published at a later date, when sufficient data (i.e. a third epoch of observations) has been obtained for the kinematical study of those sources. In Sect. 2 the observations and data reduction are described; the data analysis is outlined in Sect. 3. The results are presented and discussed in Sect. 4, and we conclude with Sect. 5. Throughout the paper we adopt the cosmological parameters as found by WMAP5 (Komatsu et al. 2009; $\left.H_{0}=70.1 \mathrm{~km} \mathrm{~s}^{-1} \mathrm{Mpc}^{-1}, \Omega_{\Lambda}=0.721, \Omega_{\mathrm{m}}=0.279\right)$.

\section{Observations and data reduction}

On March 13, 2008, we observed seven radio sources from the CORALZ sample with global VLBI at $5.0 \mathrm{GHz}$ with a bandwidth of $16 \mathrm{MHz}$, using the EVN (European VLBI Network) and the VLBA (Very Long Baseline Array). A similar observing strategy was used as for the previous global VLBI observations (de Vries et al. 2009), in order to minimize differences in signal-to-noise and resolution. Just like our observations in 2004, this global VLBI experiment was conducted with an array of 16 stations, using the EVN antennas Effelsberg, Jodrell Bank, Medicina, Noto, Torun, Westerbork, and Onsala, and the VLBA antennas St. Croix, Hancock, North Liberty, Fort Davis, Los Alamos, Pie Town, Kitt Peak, Owens Valley, and Brewster. The largest projected baselines were typically $9000 \mathrm{~km}$, resulting in a resolution of about 2 mas. Each source was typically observed for $4 \times 10 \mathrm{~min}$, spread in time to obtain optimal $U V$ coverage. 4C 39.25 and $\mathrm{J} 1657+5705$ were observed as primary calibrators. The data correlation was performed at the Joint Institute for VLBI in Europe (JIVE). The Astronomical Image Processing System (AIPS) has been used for editing, a-priori calibration, fringe-fitting, self-calibration and imaging of the data. For fringe-fitting, the AIPS task FRING was used with a point source model, a solution interval of $6 \mathrm{~min}$, and a standard signalto-noise ratio cutoff of 5. Several iterations of phase (or amplitude \& phase) self-calibration and imaging were performed using the AIPS tasks CALIB and IMAGR, until the image quality converged, with negligible negative structure and a low noise level of about $0.2 \mathrm{mJy}$.

\section{Data analysis}

The observed radio source structures were characterised using the AIPS task JMFIT, which fits (by least-squares) up to four Gaussian components to an image subsection. A first guess of the input parameters (position, flux density, and shape of the components) was made using the INPFIT procedure. To obtain error estimates of the component positions we have used the formula from Fomalont (1999):

$\sigma_{r}=\frac{\sigma_{\mathrm{rms}} d}{2 I_{\mathrm{peak}}}$

where $\sigma_{\text {rms }}$ is the post-fit rms error of the map, and $d$ and $I_{\text {peak }}$ are the size and the peak intensity of the component. The positional uncertainties were derived similarly in de Vries et al. (2009), where it was already noted that the error estimates only take into account the errors in the analysis of the reduced radio maps, not the possible biases introduced during the synthesis of these maps. This also applies here, and we will return to this issue in Sect. 4.1. The calculated hotpot separations concern the distance between the centres of the two components, not between the outer edges of the components, because the latter cannot be derived with sufficient accuracy to study the kinematics of poorly resolved components in maps with differing beam shapes.

\section{Results and discussion}

We present the third epoch of VLBI observations for two sources from the CORALZ sample, CORALZ J083139+460800, and CORALZ J131739+411545. These have also been observed as part of the VLBA Imaging and Polarimetry Survey (VIPS, Helmboldt et al. 2007). In Figs. 4 and 5 we show all maps of CORALZ J083139+460800 and CORALZ J131739+411545, respectively, including our new epoch of observations. In the following sections the results from the kinematic analyses of these sources are presented and discussed.

\subsection{CORALZ J083139+460800}

CORALZ J083139+460800 is perfectly suitable for kinematic analysis. It exhibits a compact double morphology with two strong, unresolved components separated by about 4.4 mas. We rereduced our May 2004 data for this source, since an additional (possibly spurious) component was cleaned, not detected in the other epochs, biasing the positions of the two radio lobes. The two hotspot separation measurements differ with a $3 \sigma$ significance. Note that the error estimate is based on the reduced map only, and does not include possible biases introduced during the synthesis of the map, as was noted by de Vries et al. (2009).

In Table 1 the measured component separations for CORALZ J083139+460800 are presented, with in Col. 1 the observation date, in Col. 2 the post-fit rms error of the map, in Col. 3 the measured hotspot separation in milliarcseconds, and in Col. 4 the $1-\sigma$ uncertainty of the hotspot separation. Figure 1 shows the measured component separations in milliarcseconds for CORALZ J083139+460800 from March 2000 to March 2008, with the vertical bars indicating the 1- $\sigma$ uncertainties. The data are in excellent agreement with a linear expansion.

The solid line in Fig. 1 is the result of a linear least-squares fit to the data, with a slope of $16 \pm 3$ microarcsec per year, corresponding to a lobe-to-lobe, projected expansion rate of $0.095 \pm$ $0.020 h^{-1} c=0.136 \pm 0.029 c$ at this redshift $(z=0.127)$. It is the lowest expansion speed measured in any GPS radio source so far, supporting the dependence of the expansion speeds of young radio sources on their radio luminosities. The projected expansion speed of $v_{\exp }=0.136 \pm 0.029 c$, together with the measured projected hotspot separation of 4.4 milliarcseconds, corresponding to 33 light years, yields a dynamical age for CORALZ J083139+460800 of $245 \pm 55$ years. This confirms that CORALZ J083139+460800 is a genuinely young radio 
Table 1. Hotspot separation measurements and error estimates of CORALZ J083139+460800.

\begin{tabular}{lccc}
\hline \hline observation date & $\begin{array}{c}\sigma_{\text {rms }} \\
(\mathrm{mJy})\end{array}$ & $\begin{array}{c}\text { separation } \\
(\mathrm{mas})\end{array}$ & $\begin{array}{c}\sigma_{\text {separation }} \\
(\mathrm{mas})\end{array}$ \\
\hline 2000 March 2 & 0.30 & 4.349 & 0.023 \\
2004 May 24 & 0.38 & 4.416 & 0.021 \\
2006 May 31 & 0.40 & 4.435 & 0.024 \\
2008 March 13 & 0.32 & 4.483 & 0.017 \\
\hline
\end{tabular}

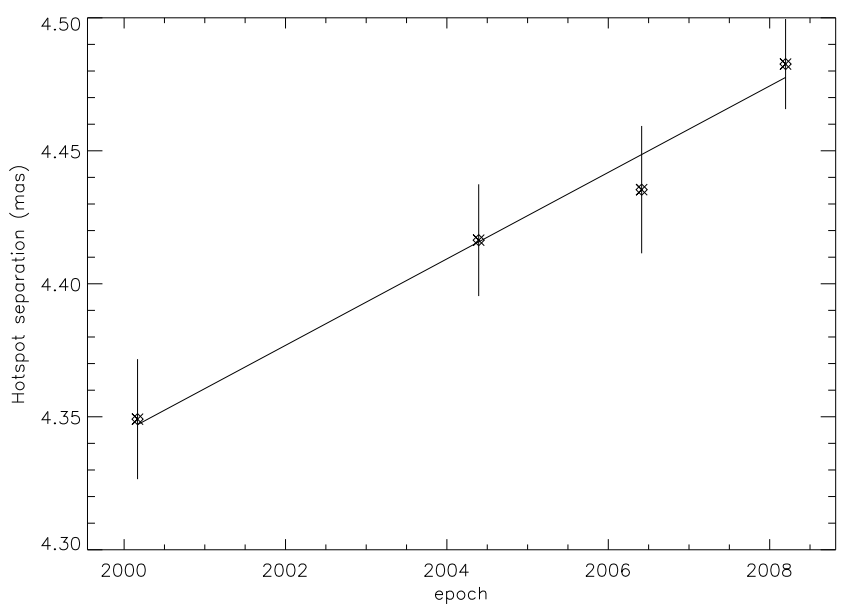

Fig. 1. The measured hotspot separations in milliarcseconds for CORALZ J083139+460800 from March 2000 to March 2008, with the vertical bars indicating the $1-\sigma$ uncertainties. The solid line is the result of a linear least-squares fit to the data, and has a slope of 16 microarcsec per year.

source, with an age similar to those found for other compact symmetric objects (e.g. Polatidis \& Conway 2003).

\subsection{CORALZ J131739+411545}

It is more of a challenge to analyse CORALZ J131739+411545, because of its complicated morphology. Its morphology was originally classified as complex (CX), but considering all data currently available (Fig. 5), we now tentatively classify this source as a compact double (CD) radio source. The problem is that the strongest component is not resolved well enough for detailed modelling of its structure, but too extended to model it as a simple Gaussian component. Furthermore it is located relatively close to the second component, making the construction of a reliable radio map and the measurement of the component positions a challenging effort. For this source JMFIT was run on each of the two components separately, because otherwise the residuals of the strongest component made the solution for the second component diverge. The derived errors are crude estimates. On the one hand they are overestimated because we estimate the centre of the components much more accurately than the values of $\sigma_{\text {rms }}$ indicate, because these are dominated by symmetric residuals on either side of the component. On the other hand the errors might be underestimated, because the error estimates do not include possible biases introduced during the synthesis of the map, which might particularly arise in sources with a complex structure like this.

In Table 2 the measured component separations for CORALZ J131739+411545 are presented, with in Col. 1 the observation date, in Col. 2 the post-fit rms error of the subimage containing the strongest component, in Col. 3 the post-fit
Table 2. Hotspot separation measurements and error estimates of CORALZ J131739+411545.

\begin{tabular}{lcccc}
\hline \hline observation date & $\begin{array}{c}\sigma_{\text {rms,a }} \\
(\mathrm{mJy})\end{array}$ & $\begin{array}{c}\sigma_{\text {rms,b }} \\
(\mathrm{mJy})\end{array}$ & $\begin{array}{c}\text { separation } \\
(\mathrm{mas})\end{array}$ & $\begin{array}{c}\sigma_{\text {separation }} \\
(\mathrm{mas})\end{array}$ \\
\hline 2000 March 2 & 2.11 & 0.47 & 3.320 & 0.051 \\
2004 May 24 & 1.79 & 0.33 & 3.265 & 0.046 \\
2008 March 13 & 2.05 & 0.14 & 3.466 & 0.065 \\
\hline
\end{tabular}

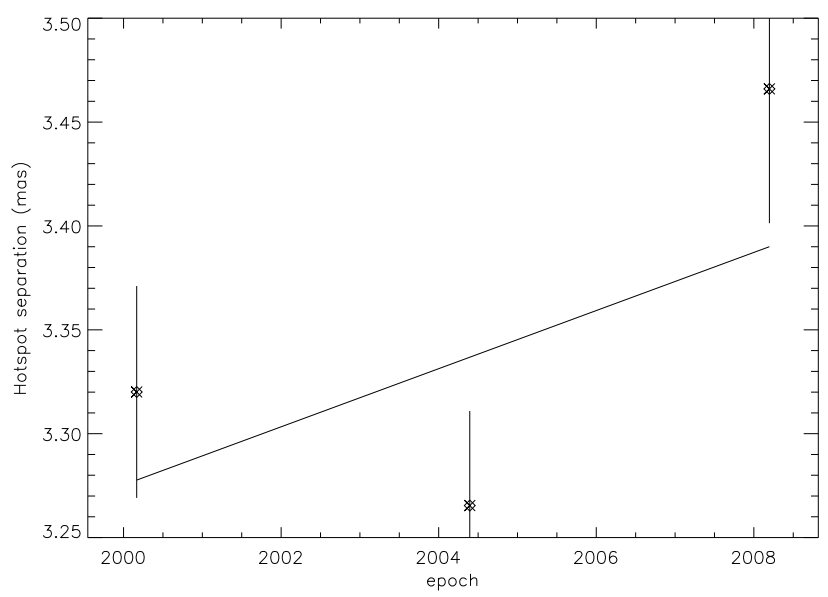

Fig. 2. The measured hotspot separations in milliarcseconds for CORALZ J131739+411545 from March 2000 to March 2008, with the vertical bars indicating the $1-\sigma$ uncertainties. The solid line is the result of a linear least-squares fit to the data, and has a slope of $14 \pm$ 10 microarcsec per year.

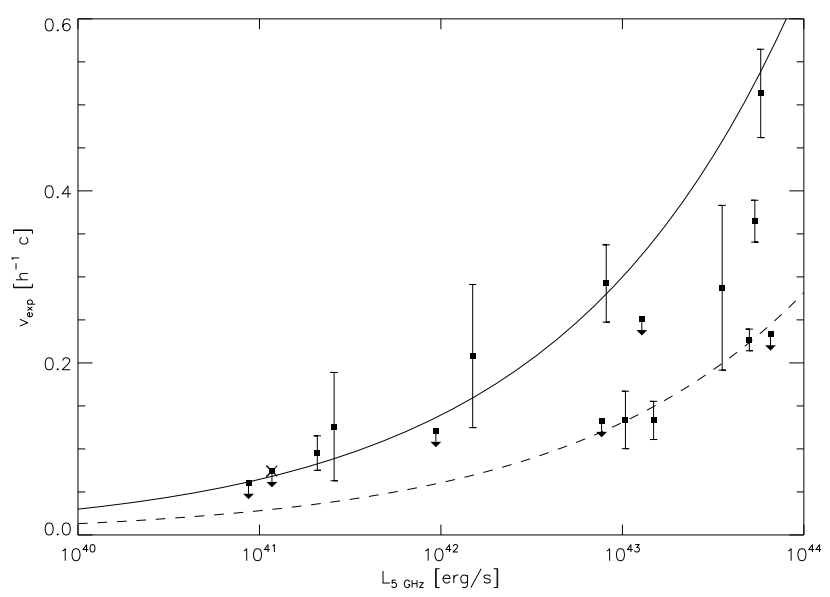

Fig. 3. Expansion velocities of compact symmetric objects in units of $h^{-1} c$, and radio luminosities at $5 \mathrm{GHz}$ in $\mathrm{erg} \mathrm{s}^{-1}$. Arrows indicate upper limits. The one indicated with the cross is the upper limit to the expansion velocity of CORALZ J131739+411545 presented in this paper. The solid line shows the expected relation between expansion velocity and radio luminosity, derived from analytic radio source evolution modelling (de Vries et al. 2009), with arbitrary scaling. The dashed line represents this relation scaled down by a factor of $\sqrt{1-0.9^{2}} \simeq 0.44$, such that $90 \%$ of a sample of randomly oriented sources that intrinsically would follow the solid line, should be located above the dashed line.

rms error of the subimage of the second component, in Col. 4 the measured hotspot separation in milliarcseconds, and in Col. 5 the $1-\sigma$ uncertainty of the hotspot separation. Figure 2 shows the measured hotspot separations in milliarcseconds for CORALZ J131739+411545 from March 2000 to March 2008, with the vertical bars indicating the $1-\sigma$ uncertainties. From this 


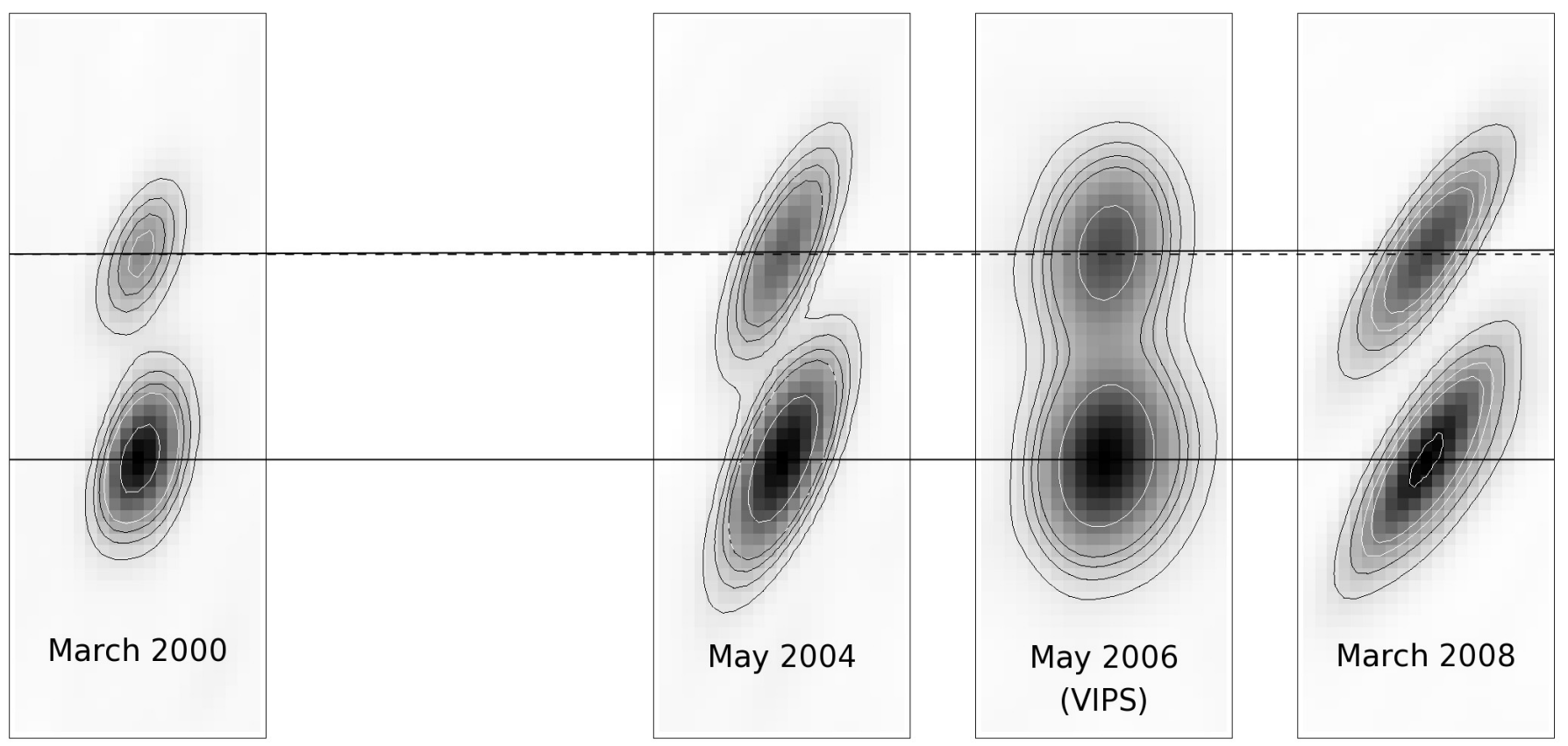

Fig. 4. Contour maps of CORALZ J083139+460800, observed in March 2000, in May 2004, in May 2006 with the VLBA as part of VIPS (Helmboldt et al. 2007), and in March 2008. Peak flux densities of each map are: 31.7, 38.9, 44.4, and 26.8 mJy. The rms noise levels are: 0.14 , $0.14,0.26$, and $0.06 \mathrm{mJy}$. Contours are drawn at 3, 6, 9, 12, and $24 \mathrm{mJy}$. All maps have been aligned and rotated, such that the (lower) solid line, running through the main component's centre in each map, is (horizontal). The dashed line runs parallel to this line, while the (upper) solid line runs through the secondary component's centre in each map. The lines slowly diverge, revealing a small but significant increase in the separation between the two components. This increase corresponds to a projected hotspot separation velocity of $0.095 \pm 0.020 \mathrm{~h}^{-1} c$, yielding a dynamical age of $245 \pm 55$ years. Note that both components are unresolved, and therefore the varying component shapes arise from a varying clean beam shape, not from changes in the source structure.

figure the error estimates appear reasonable. We perform a linear least-squares fit to the data, resulting in a slope of $14 \pm$ 10 microarcsec per year, which corresponds to a 1- $\sigma$ upper limit to the projected expansion rate of $0.074 h^{-1} c=0.106 c$ at a redshift of $z=0.066$ for CORALZ J131739+411545. This limit, together with the measured projected hotspot separation of 3.3 milliarcsec, corresponding to 14 light years, yields a lower limit to the dynamical age of CORALZ J131739+411545 of 130 years. This result is in good agreement with the upper limit of 4300 years, derived from the spectral ageing analysis of CORALZ J131739+411545 (Mack et al. 2009).

\subsection{Expansion velocity and radio power}

The new results described above significantly increase the sparsely available data on expansion velocities of young radio sources. It is therefore important to review the correlation between expansion speed and luminosity found by de Vries et al. (2009). The expansion speed we determine here for CORALZ J083139+460800, exceeds the previously derived upper limit, but is still the lowest expansion speed measured in any GPS radio source so far. Being a relatively weak radio source $\left(L_{5} \mathrm{GHz}=10^{41.3} \mathrm{erg} \mathrm{s}^{-1}\right.$, compared to a median radio luminosity of $L_{5 \mathrm{GHz}}=10^{43.2} \mathrm{erg} \mathrm{s}^{-1}$ for sources with previously determined expansion speeds), this result supports the correlation between the expansion speed and radio luminosity. Further support comes from CORALZ J131739+411545 $\left(L_{5} \mathrm{GHz}=10^{41.1} \mathrm{erg} \mathrm{s}^{-1}\right)$, with an upper limit to the expansion speed lower than the value found for any radio source with a higher radio luminosity.
In Fig. 3 we show the expansion velocities measured in young radio sources as a function of radio luminosity. Both the radio luminosity and the lobe-to-lobe, projected expansion velocity have been corrected for cosmological effects, and represent rest-frame quantities. The arrows indicate upper limits. The one indicated with the cross is the upper limit to the expansion velocity of CORALZ J131739+411545 presented in this paper. In de Vries et al. (2009) we presented an analytic model of a young, expanding radio source with a constant jet power in a power-law density environment. This model predicts that $v_{\text {exp }} \propto L_{\text {radio }}^{1 / 3}$. The solid line represents such a relation, with arbitrary scaling. The dashed line represents this relation scaled down by a factor of $\sqrt{1-0.9^{2}} \simeq 0.44$, such that $90 \%$ of a sample of randomly oriented sources that intrinsically would follow the solid line, should be located above the dashed line. A Spearman rank correlation analysis shows that, including the measured expansion speed presented in this paper and ignoring all upper limits, the correlation is significant at a $99 \%$ confidence level ( $n=10, \rho=0.794)$. Including the upper limits in the statistical analysis tends to increase the significance of the correlation, regardless of the precise way these upper limits are treated, since the upper limits of the low-luminosity radio sources are so stringent. Although it is still too early to draw definite conclusions concerning the deviations of the data from the model, it seems the data indicate a relation less steep than the analytic model, suggesting the density $\rho$ of the radio source environment is shallower than the assumed power-law slope of $\beta=2$ $\left(\rho \propto r^{-\beta}\right)$, since the exponent of the radio luminosity $L_{\text {radio }}$ in this relation depends on the power-law slope of the environment as $1 /(5-\beta)$ (de Vries et al. 2009). The random orientation of 
N. de Vries et al.: The dynamical ages of two low-luminosity young radio sources
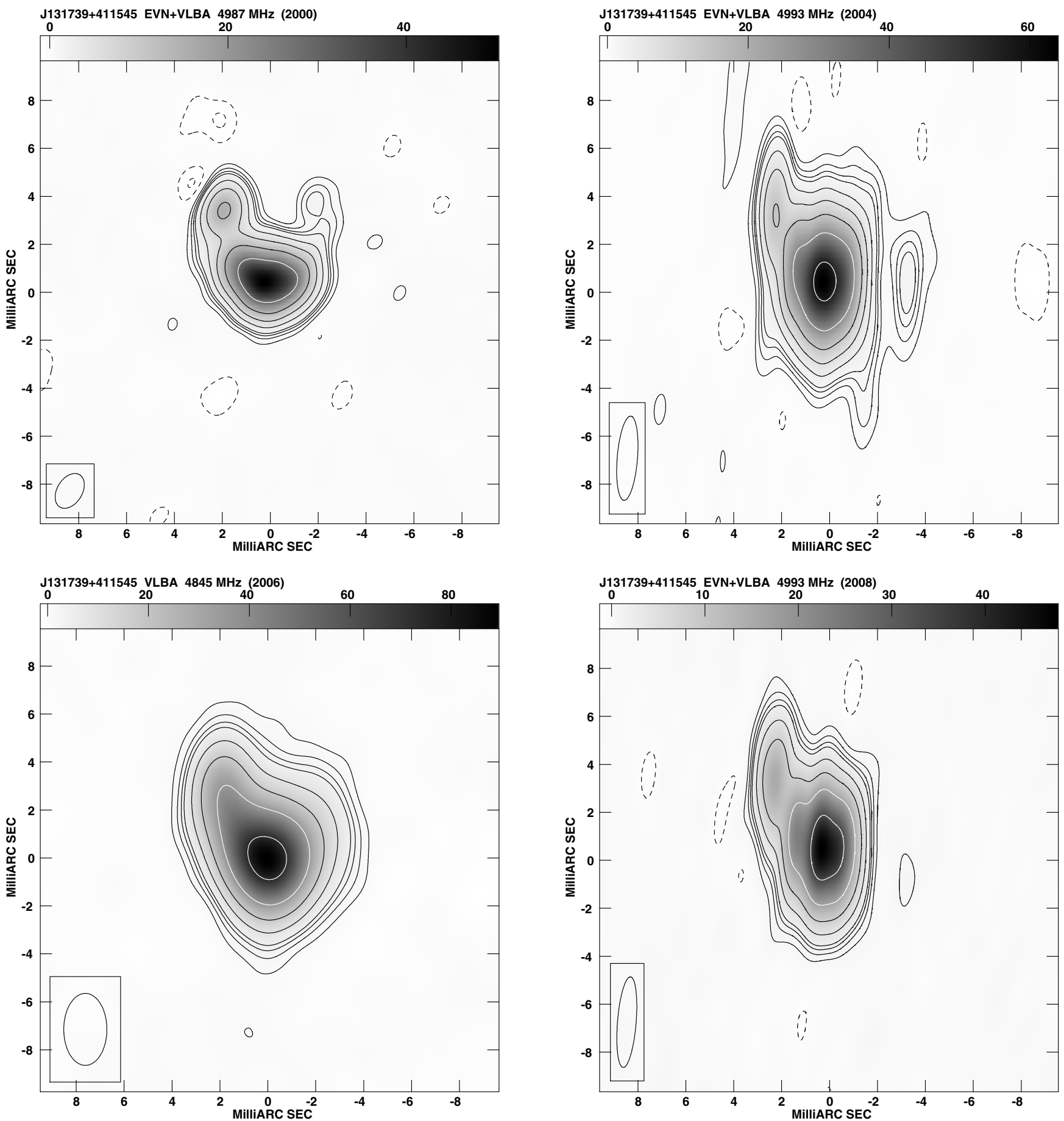

Fig. 5. Contour maps of CORALZ J131739+411545, observed in March 2000 (top left), in May 2004 (top right), in May 2006 with the VLBA as part of VIPS (Helmboldt et al. 2007; bottom left), and in March 2008 (bottom right). Peak flux densities of each map are: 50.3, 63.8, 89.3, and $47.7 \mathrm{mJy}$. The rms noise levels are: $0.14,0.14,0.37$, and $0.17 \mathrm{mJy}$. Contours are drawn at $(-6,-3,3,6,9,12,24,48,96,200,400,800$, 1600) times the rms noise level of the radio map. Negative contours are indicated with dashed lines.

the radio sources only introduces a vertical scatter, and does not affect the slope of the correlation.

\section{Conclusions}

In this paper we present a new epoch of global VLBI observations of sources from the CORALZ sample. For CORALZ J083139+460800 this resulted in the detection of its projected hotspot separation velocity of $0.095 \pm 0.020 \mathrm{~h}^{-1} c$, representing the lowest expansion speed measured so far. This result, together with the measured hotspot separation, yields a dynamical age of $245 \pm 55$ years. Despite the complicated structure of the double radio source CORALZ J131739+411545, we were able to derive an upper limit to the expansion speed of this source of $0.074 h^{-1} c$, yielding a lower limit for the dynamical age of 130 years. Both expansion speeds are very low compared to CSOs with higher radio luminosities, confirming the dependence of the expansion speeds of young radio sources on their radio luminosities. These new measurements raise the significance of the correlation above $99 \%$, and indicate the slope 
of this relation might be less steep than our model, suggesting the radio source environments are shallower than $\rho \propto r^{-2}$.

Acknowledgements. The European VLBI Network is a joint facility of European, Chinese, South African and other radio astronomy institutes funded by their national research councils (proposal codes: GS016, GS021, GV019). The National Radio Astronomy Observatory is a facility of the National Science Foundation operated under cooperative agreement by Associated Universities, Inc (proposal codes: GS016, GS021, GV019).

\section{References}

Conway, J. E. 2002, New Astron. Rev., 46, 263

de Vries, N., Snellen, I. A. G., Schilizzi, R. T., Mack, K.-H., \& Kaiser, C. R. 2009, A\&A, 498, 641

Fanti, C., \& Fanti, R. 2003, Radio Astronomy at the Fringe, ed. J. A. Zensus, M. H. Cohen, \& E. Ros, 300, 81

Fanti, R., Fanti, C., Schilizzi, R. T., et al. 1990, A\&A, 231, 333

Fomalont, E. B. 1999, Synthesis Imaging in Radio Astronomy II, 180, 301

Helmboldt, J. F., Taylor, G. B., Tremblay, S., et al. 2007, ApJ, 658, 203
Komatsu, E., Dunkley, J., Nolta, M. R., et al. 2009, ApJS, 180, 330

Mack, K.-H., Snellen, I. A. G., Schilizzi, R. T., \& de Vries, N. 2009, Astron. Nachr., 330, 217

McMahon, R. G., \& Irwin, M. J. 1991, in Proc. Conf. Digitised Optical Sky Surveys, ed. H. T. MacGillivray, \& E. B. Thomson (Dordrecht: Kluwer Acad. Publ.), 417

O’Dea, C. P. 1998, PASP, 110, 493

O'Dea, C. P., \& Baum, S. A. 1996, in GPS and CSS radio sources, ed. I. A. G. Snellen, R. T. Schilizzi, H. J. A. Röttgering, \& M. N. Bremer, 241

Owsianik, I., \& Conway, J. E. 1998, A\&A, 337, 69

Peacock, J. A., \& Wall, J. V. 1982, MNRAS, 198, 843

Polatidis, A. G., \& Conway, J. E. 2003, Publ. Astron. Soc. Aust., 20, 69

Readhead, A. C. S., Taylor, G. B., Xu, W., et al. 1996, ApJ, 460, 612

Snellen, I. A. G., Schilizzi, R. T., Miley, G. K., et al. 2000, MNRAS, 319, 445

Snellen, I. A. G., Mack, K.-H., Schilizzi, R. T., \& Tschager, W. 2003, Publ. Astron. Soc. Aust., 20, 38

Snellen, I. A. G., Mack, K.-H., Schilizzi, R. T., \& Tschager, W. 2004, MNRAS, 348,227

White, R. L., Becker, R. H., Helfand, D. J., \& Gregg, M. D. 1997, ApJ, 475, 479

Wilkinson, P. N., Polatidis, A. G., Readhead, A. C. S., Xu, W., \& Pearson, T. J. 1994, ApJ, 432, L87 